\title{
Small Dense Low-Density Lipoprotein for Risk Prediction of Acute Coronary Syndrome
}

\author{
Abhishek Singh ${ }^{\mathrm{a}}$, Rajendra Puhan ${ }^{\mathrm{a}}$, Akshyaya Pradhan $^{\mathrm{b}, \mathrm{d}}{ }^{\mathrm{d}}$, Wahid Alic ${ }^{\mathrm{c}}$, Rishi Sethi ${ }^{\mathrm{b}}$
}

\begin{abstract}
Background: Elevated Low density Lipoprotein (LDL) levels have been traditionally associated with development of atherosclerotic coronary artery disease (CAD). As LDL-cholesterol levels are not always raised in acute coronary syndrome (ACS) patients, sd-LDL is an emerging risk factor. The purpose of the present study was tri-fold. Firstly, it aimed to investigate the association of sd-LDL as a risk factor in ACS patients. Secondly, it aimed to correlate the presence of sdLDL with severity of coronary artery disease as determined by coronary angiography. Lastly, it aimed to correlate the presence of sd-LDL with short-term outcomes.
\end{abstract}

Methods: This was a prospective, hospital-based, cross-sectional, case-control study conducted over a 1-year study duration. Patients above the age of 18 years diagnosed with ACS within $24 \mathrm{~h}$ of admission were studied. Blood samples were collected after all patients had undergone coronary angiography and sd-LDL levels were measured.

Results: ACS patients had significantly higher sd-LDL levels than non-ACS patients $(16.10 \pm 1.42 \mathrm{mg} / \mathrm{dL}$ vs. $12.67 \pm 0.71 \mathrm{mg} / \mathrm{dL}, \mathrm{P}=$ $0.036)$. Males had significantly higher sd-LDL levels than females $(16.79 \pm 1.55 \mathrm{mg} / \mathrm{dl}$ vs. $10.77 \pm 2.62 \mathrm{mg} / \mathrm{dl}, \mathrm{P}=0.047)$. Patients with non-left anterior descending coronary artery (LAD) involvement had significantly higher sd-LDL levels compared to patients with LAD involvement $(21.92 \pm 3.55 \mathrm{mg} / \mathrm{dL}$ vs. $14.03 \pm 1.35 \mathrm{mg} / \mathrm{dL}, \mathrm{P}=0.007)$.

Conclusion: These results suggest that sd-LDL is a risk factor for the development of ACS in an Indian population.

Keywords: Acute coronary syndrome; Coronary artery disease; Left anterior descending coronary artery; Major adverse cardiac events;

Manuscript submitted March 17, 2021, accepted April 27, 2021

Published online July 9, 2021

aDepartment of Internal Medicine, King George's Medical University, Lucknow, Uttar Pradesh 226003, India

bDepartment of Cardiology, King George's Medical University, Lucknow, Uttar Pradesh 226003, India

'Department of Pathology, King George's Medical University, Lucknow, Uttar Pradesh 226003, India

${ }^{\mathrm{d} C}$ Corresponding Author: Akshyaya Pradhan, Department of Cardiology, King George's Medical University, Lucknow, Uttar Pradesh 226003, India.

Email: akshyaya33@gmail.com

doi: https://doi.org/10.14740/cr1254
Small density low-density lipoprotein

\section{Introduction}

Low-density lipoprotein (LDL) is the key driver of atherogenesis [1]. It is the predominant cholesterol-carrier in the bloodstream and is therefore the principal target for prevention of coronary artery disease (CAD). This lipoprotein is a composite of heterogeneous particles that differ in size, density, and chemical composition. It has two phenotypes: pattern A consists primarily of large buoyant LDL (lb-LDL) particles and pattern B consists primarily of small dense LDL (sd-LDL) particles. Sd-LDL particles are more atherogenic due to increased arterial wall penetration, less binding affinity for the LDL receptor, prolonged plasma half-life, and less oxidative stress resistance compared to lb-LDL [2]. However, LDL concentration is not always raised in patients with acute coronary syndrome (ACS); thus, sd-LDL may be an emerging risk factor in ACS patients [3].

Data exploring the relationship of sd-LDL particles with ACS in an Indian population are scarce. Hence, this study was conducted to: 1) investigate the association of sd-LDL as a risk factor in ACS patients; 2) correlate the presence of sd-LDL with severity of CAD as determined by coronary angiography; and 3) to correlate the presence of sd-LDL with short-term outcomes.

\section{Materials and Methods}

\section{Study design}

This was a prospective, hospital-based, cross-sectional, casecontrol study conducted between August 2015 and August 2016. Consecutive patients who were admitted to either the Emergency Department of Medicine or Cardiology Department above the age of 18 years with a diagnosis of ACS within $24 \mathrm{~h}$ of admission (prior to taking first dose of statins) were enrolled in this study. Patients with chronic renal failure, liver cirrhosis, pregnancy, malignancy, prior statin therapy, or $\leq 1$-year prior ACS were excluded from the study. Cases were defined as patients admitted with a diagnosis of ACS that had fulfilled the inclusion criteria. Controls were defined as age- and sexmatched healthy individuals. The study enrolled 70 healthy 
controls and 75 cases. The study protocol was approved by and conducted in compliance with the ethical standards of the Institutional Ethics committee as with the Helsinki Declaration. All patients provided written informed consent for study participation prior to the commencement of the study.

\section{Study definitions}

ACS was defined as any group of clinical symptoms compatible with acute myocardial ischemia covering the spectrum of clinical conditions ranging from unstable angina (UA) to nonST-segment elevation myocardial infarction (NSTEMI) to STsegment elevation myocardial infarction (STEMI). Acute myocardial infarction was diagnosed by detection of rise and/or fall of cardiac biomarker values with at least one value above the 99th percentile above upper reference limit with at least one of the following: 1) symptoms of ischemia; 2) new or presumed new significant ST segment, T wave changes or new left bundle branch block (LBBB); 3) development of pathological Q waves on electrocardiography; 4) imaging evidence of new loss of viable myocardium or new regional wall motion abnormality; or 5) identification of an intracoronary thrombus by angiography. CAD was defined as stenosis of one or more coronary artery branch with $\geq 50 \%$ diameter luminal narrowing as observed on coronary angiography. Patients were categorized according to involvement or non-involvement of the left anterior descending coronary artery (LAD). UA was usually considered when cardiac biomarkers were undetectable in the bloodstream hours after initial onset of ischemic pain. It presents with one or more of the following characteristics: 1) rest angina (usually lasting $>$ $20 \mathrm{~min}$ ); 2) new-onset (less than 2 months prior) severe angina; and 3 ) a crescendo pattern of occurrence (increasing in intensity, duration, frequency, or any combination of these factors.

\section{Study procedure}

Blood samples were obtained by venipuncture after the patient had undergone coronary angiography. The serum samples were separated and stored at $-20{ }^{\circ} \mathrm{C}$ within $1 \mathrm{~h}$ of taking the sample. The method of sd-LDL and lb-LDL isolation from serum was based on a two-step procedure reported by Hirano et al in 2004 [4]. Precipitation reagent $(0.1 \mathrm{~mL})$ containing $150 \mathrm{U} / \mathrm{mL}$ heparin sodium salt and $90 \mathrm{mM} / \mathrm{L} \mathrm{MgCl}_{2}$ was added to $0.1 \mathrm{~mL}$ serum sample. After mixing, the sample was incubated at 37 ${ }^{\circ} \mathrm{C}$ for $10 \mathrm{~min}$. Next, each sample was incubated in an ice bath for $15 \mathrm{~min}$ and centrifuged at $15,000 \mathrm{rpm}$ for $15 \mathrm{~min}$ at $4{ }^{\circ} \mathrm{C}$. The supernatant containing sd-LDL was carefully removed and saved. The pellet containing lb-LDL was dissolved in $0.1 \mathrm{M}$ sodium phosphate buffer containing $0.9 \% \mathrm{NaCl}$ at $\mathrm{pH}$ 7.4. Appropriate aliquots of LDL, sd-LDL, and lb-LDL fraction were used for analysis of their cholesterol and apoprotein B content.

\section{Validation of sd-LDL assay}

Sd-LDL assay in serum sample was performed with a com- mercially available kit (Randox Laboratories, Crumlin, UK) as per kit insert. Validation of sd-LDL test procedure was carried out by testing quality control materials of known concentration. The quality control data obtained were reproducible as per reported value of sd-LDL concentration. Three levels of control were used to observe precision and accuracy of the test procedure as well as verification of the test procedure. Further, Randox Laboratories has validated the test procedure by ultracentrifugation and clearance method of sd-LDL-C kit available in the market and observed good correlation.

\section{Study outcomes}

Study outcomes were major adverse cardiovascular events (MACE), in-hospital, and 30-day mortality. MACE was defined as a composite of cardiac death, cardiogenic shock, acute left ventricular failure, recurrent myocardial infarction, hemodynamically unstable arrhythmia, prolonged anginal pain, and conditions necessitating immediate coronary intervention.

\section{Data collection}

Data such as age, gender, cardiovascular risk factors, cardiovascular history, clinical presentation, affected lesions, and ongoing medications were collected from patients at initial evaluation.

\section{Statistical analysis}

Continuous data are expressed as mean \pm standard error. Categorical data are expressed as numbers and percentages. Groups were compared by Student's $t$-test. Groups were also compared by one-way analysis of variance and the significance of mean difference between the groups was done by Tukey's honestly significant difference post-hoc test after ascertaining normality by Shapiro-Wilk's test and homogeneity of variance by Levene's test. A two-tailed P value $<0.05$ was considered statistically significant. All statistical analysis was done using Statistical Package for Social Sciences (SPSS, Chicago, IL, USA) program, version 17.

\section{Results}

\section{Demographic characteristics of cases}

The study comprised 75 cases and 70 age- and sex-matched controls. The mean age of the cases was $52.02 \pm 1.16$ years. Almost half of the cases, i.e., 37 (49.3\%), were below the age of 50 years. Males comprised $54(72.0 \%)$ of the cases. Forty-five $(60.0 \%)$ cases had body mass index $(\mathrm{BMI})>24 \mathrm{~kg} / \mathrm{m}^{2}$. Conventional risk factors were present only in $48(64.0 \%)$ cases. Of the 75 cases, $42(56.0 \%)$ were smokers, 25 (33.3\%) were hypertensives, and $27(36.0 \%)$ were diabetics. More than three-quarters cases, i.e. $58(77.3 \%)$, presented with STEMI. The angiographic outcome was single-vessel disease in 41 (54.7\%) patients, dou- 
Table 1. Demographic Characteristics of Cases

\begin{tabular}{|c|c|}
\hline Characteristic & $\begin{array}{l}\text { Patients } \\
(n=75)\end{array}$ \\
\hline Age, years & $52.02 \pm 1.16$ \\
\hline$<50$ & $37(49.3 \%)$ \\
\hline $50-60$ & $23(30.7 \%)$ \\
\hline$>60$ & $15(20.0 \%)$ \\
\hline Male & $54(72.0 \%)$ \\
\hline \multicolumn{2}{|l|}{ Body mass index } \\
\hline$\leq 24 \mathrm{~kg} / \mathrm{m}^{2}$ & $30(40.0 \%)$ \\
\hline$>24 \mathrm{~kg} / \mathrm{m}^{2}$ & $45(60.0 \%)$ \\
\hline Conventional risk factors & $48(64.0 \%)$ \\
\hline Smoker & $42(56.0 \%)$ \\
\hline Hypertension & $25(33.3 \%)$ \\
\hline Diabetes mellitus & $27(36.0 \%)$ \\
\hline Family history of coronary artery disease & $8(10.7 \%)$ \\
\hline \multicolumn{2}{|l|}{ Diagnosis } \\
\hline Non-ST-segment elevation myocardial infarction & $17(22.7 \%)$ \\
\hline ST-segment elevation myocardial infarction & $58(77.3 \%)$ \\
\hline \multicolumn{2}{|l|}{ Angiographic outcome } \\
\hline Single-vessel disease & $41(54.7 \%)$ \\
\hline Double-vessel disease & $13(17.3 \%)$ \\
\hline Triple-vessel disease & $21(28.0 \%)$ \\
\hline \multicolumn{2}{|l|}{ Involvement of coronary artery } \\
\hline Left anterior descending coronary artery & $59(78.7 \%)$ \\
\hline Non-left anterior descending coronary artery & $16(21.3 \%)$ \\
\hline
\end{tabular}

Values are expressed as mean \pm standard deviation or number (percent).

ble-vessel disease in $13(17.3 \%)$ patients, and triple-vessel disease in $21(28.0 \%)$ patients. The involved artery was LAD in 59 $(78.7 \%)$ cases and non-LAD in $16(21.3 \%)$ cases. Demographic characteristics of the cases are detailed in Table 1.

\section{Sd-LDL levels of controls and cases}

Sd-LDL levels were significantly higher in cases compared to controls $(16.10 \pm 1.42 \mathrm{mg} / \mathrm{dL}$ vs. $12.67 \pm 0.71 \mathrm{mg} / \mathrm{dL}, \mathrm{P}=$ $0.036)$ as displayed in Figure 1.

\section{Sd-LDL levels of cases}

Sd-LDL levels decreased as age increased $(<50$ years: 17.04 $\pm 2.47 \mathrm{mg} / \mathrm{dL}, 50-60$ years: $16.84 \pm 2.67 \mathrm{mg} / \mathrm{dL},>60$ years: $13.53 \pm 1.62 \mathrm{mg} / \mathrm{dL})$. However this inverse relation was not statistically significant. Males had significantly higher sd-LDL levels than females $(16.79 \pm 1.55 \mathrm{mg} / \mathrm{dL}$ vs. $10.77 \pm 2.62 \mathrm{mg} /$ $\mathrm{dL}, \mathrm{P}=0.047)$. Smokers, hypertensives, and diabetics had higher sd-LDL levels compared to non-smokers, normoten-
20.0 $\mathrm{p}=0.036$

16.0 $12.67 \pm 0.71$

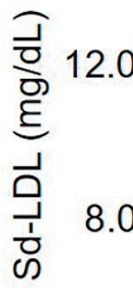

4.0

0.0

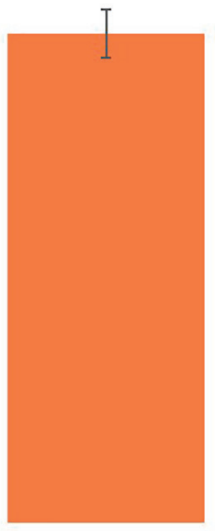

$16.10 \pm 1.42$

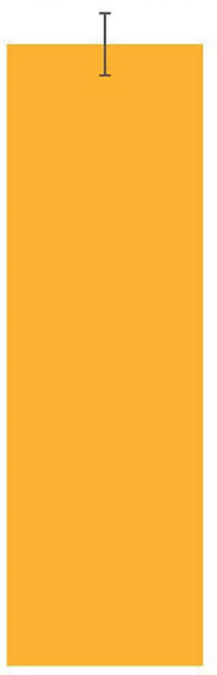

Controls $\backsim$ Cases

Figure 1. Sd-LDL levels of controls and cases. sd-LDL: small density low-density lipoprotein.

sives, and non-diabetics although not statistically significant. STEMI patients had higher sd-LDL levels than NSTEMI patients. Angiographic outcome did not indicate any association or display any trend with sd-LDL. Patients with involvement of non-LAD had significantly higher sd-LDL levels compared to patients with LAD involvement $(21.92 \pm 3.55 \mathrm{mg} / \mathrm{dL}$ vs. $14.03 \pm 1.35 \mathrm{mg} / \mathrm{dL}, \mathrm{P}=0.007)$. Sd-LDL levels of the cases are elaborated in Table 2 and involvement of LAD and non-LAD is illustrated in Figure 2.

\section{Short-term outcomes of cases}

MACE occurred in two $(2.67 \%)$ patients. These patients suffered from left ventricular failure and ventricular arrhythmia, respectively. In-hospital mortality occurred in two $(2.67 \%)$ patients, whilst 30-day mortality was observed in none of the patients. Short-term outcomes are summarized in Table 3.

\section{Discussion}

Several earlier studies have shed light on the association between sd-LDL particles and ACS. The present study conducted on an Indian population confirmed higher sd-LDL levels in ACS patients $(16.10 \pm 1.42 \mathrm{mg} / \mathrm{dL})$ compared to healthy controls $(12.67 \pm 0.71 \mathrm{mg} / \mathrm{dL})$. In line with these findings, Emadzadeh et al [1] revealed higher sd-LDL levels for myocardial infarction patients $(51 \mathrm{mg} / \mathrm{dL})$ and UA patients $(46 \mathrm{mg} /$ 
Table 2. Mean sd-LDL Levels $(\mathrm{mg} / \mathrm{dL})$ of Cases

\begin{tabular}{|c|c|c|}
\hline Characteristic & Patients $(n=75)$ & P value \\
\hline \multicolumn{3}{|l|}{ Age } \\
\hline$<50$ & $17.04 \pm 2.47(n=37)$ & 0.416 \\
\hline$>60$ & $13.53 \pm 1.62(\mathrm{n}=15)$ & \\
\hline \multicolumn{3}{|l|}{ Gender } \\
\hline Female & $10.77 \pm 2.62(\mathrm{n}=21)$ & \\
\hline \multicolumn{3}{|l|}{ Body mass index } \\
\hline$\leq 24 \mathrm{~kg} / \mathrm{m}^{2}$ & $15.34 \pm 1.66(n=30)$ & 0.634 \\
\hline$>24 \mathrm{~kg} / \mathrm{m}^{2}$ & $16.84 \pm 2.31(n=45)$ & \\
\hline \multicolumn{3}{|l|}{ Conventional risk factors } \\
\hline Normotensives & $15.89 \pm 1.64(\mathrm{n}=50)$ & \\
\hline Diabetics & $13.82 \pm 2.31(n=27)$ & 0.311 \\
\hline Non-diabetics & $16.72 \pm 1.69(n=48)$ & \\
\hline \multicolumn{3}{|l|}{ Diagnosis } \\
\hline Non-ST-segment elevation myocardial infarction & $15.03 \pm 2.05(\mathrm{n}=17)$ & 0.674 \\
\hline ST-segment elevation myocardial infarction & $16.52 \pm 1.81(\mathrm{n}=58)$ & \\
\hline \multicolumn{3}{|l|}{ Angiographic outcome } \\
\hline Single-vessel disease & $16.08 \pm 1.88(n=41)$ & 0.087 \\
\hline
\end{tabular}

Values are expressed as mean \pm standard deviation. sd-LDL: small density low-density lipoprotein.

dL) compared to healthy controls $(13 \mathrm{mg} / \mathrm{dL})$. Fukushima et al [5] showed higher sd-LDL levels for ACS patients $(30 \pm 14$ $\mathrm{mg} / \mathrm{dL})$ compared to healthy controls $(22 \pm 8 \mathrm{mg} / \mathrm{dL})$. Wu et al [6] observed higher sd-LDL levels for UA patients (male: $1.09 \mathrm{mmol} / \mathrm{L}$ and female: $1.03 \mathrm{mmol} / \mathrm{L})$, STEMI patients (male: $1.30 \mathrm{mmol} / \mathrm{L}$ and female: $1.17 \mathrm{mmol} / \mathrm{L}$ ), and NSTEMI patients (male: $1.44 \mathrm{mmol} / \mathrm{L}$ and female: $1.14 \mathrm{mmol} / \mathrm{L}$ ) compared to healthy controls (male: $0.72 \mathrm{mmol} / \mathrm{L}$ and female: 0.62 $\mathrm{mmol} / \mathrm{L}$ ). These findings are suggestive of sd-LDL as a risk factor for ACS. However, ethnical variation, differing methodology, and varying cut-off points should be taken into consideration when attempting to compare precise sd-LDL levels [7-9]. Additionally, Wu et al [6] also exposed a gender effect. Sd-LDL levels were higher in males $(0.71 \pm 0.24 \mathrm{mmol} / \mathrm{L})$ compared to females $(0.63 \pm 0.22 \mathrm{mmol} / \mathrm{L})$. This observation is in agreement with our study which reported increased sdLDL levels in males $(16.79 \pm 1.55 \mathrm{mg} / \mathrm{dL})$ compared to females $(10.77 \pm 2.62 \mathrm{mg} / \mathrm{dL})$ indicative of greater male susceptibility to develop ACS. These values are all increasingly high compared to sd-LDL levels of $8.5 \pm 3.9 \mathrm{mg} / \mathrm{dL}$ reported in healthy controls belonging to an Indian population [10].

Few studies have proven the clinical significance of sd-
LDL in the assessment of CAD risk by employing various methods. Moon et al [11] reported higher fraction of sd-LDL particles in CAD patients. Kwon et al [12] fortified this finding and confirmed sd-LDL fraction to be an independent risk factor for CAD. Koba et al [13] demonstrated the association between high sd-LDL levels and CAD severity using Gensini scores. Goel et al [10] correlated sd-LDL levels and CAD severity using the SYNTAX score. The unique contribution observation of the present study is higher sd-LDL levels in CAD patients with non-LAD involvement compared to LAD involvement. This hints towards influence of sd-LDL levels on vessel involvement.

Several earlier studies have demonstrated the relationship between sd-LDL and CAD [14-18]. However, few others have failed to do so. The disparity in study findings is attributable to a few aspects. Small sample sizes with short follow-ups prove insufficient to determine correlation [2]. Racial and ethnical disparities influence clinical outcomes [13]. Different laboratory procedures have been employed to separate LDL subclasses. However, none of these have been established as a gold standard [19]. Furthermore, heparin may be administered to ACS patients. Heparin may disrupt the lipoprotein profiles of samples 


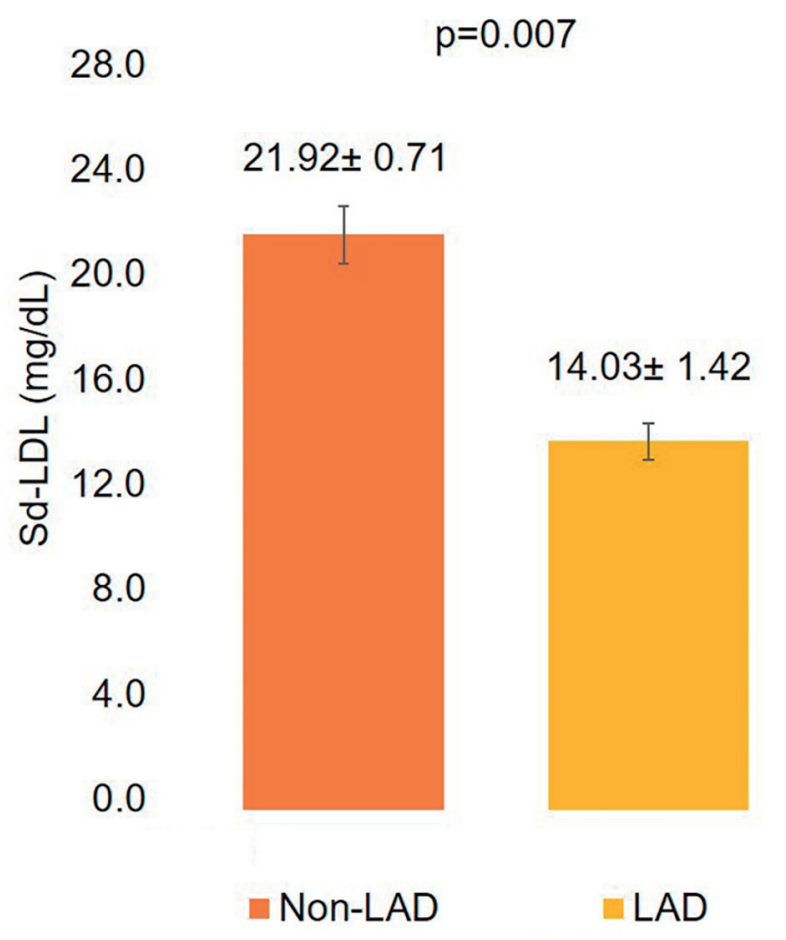

Figure 2. Comparison of sd-LDL levels according to involvement of LAD and non-LAD coronary arteries. LAD: left anterior descending coronary artery.

obtained $24 \mathrm{~h}$ following ACS [3]. Sd-LDL levels exhibit circadian changes in both diabetic patients and controls. This finding significantly impacts study findings as diabetics constitute a considerable proportion of study populations. Lastly, possible confounding factors may skew results [20].

\section{Study limitations}

The present study has a few limitations. Firstly, the sample size was small. Secondly, confounding factors were not taken into account. Thirdly, among earlier Indian studies [21-23], the Indian Council of Medical Research-India Diabetes (ICMRINDIAB) Study [24] also reported low levels of high-density lipoprotein (HDL). Hence, we did not aim to study this lipoprotein parameter.

\section{Conclusion}

Higher sd-LDL levels in cases compared to controls suggest that sd-LDL levels may serve as a potential risk factor for ACS. Higher levels of sd-LDL levels observed in males compared to females suggest that males may be more prone to develop ACS than females. Higher levels of this lipoprotein subclass were observed in patients with non-LAD involvement than patients with LAD involvement. This shows that sd-LDL levels have an effect on angiographic outcome (type of vessel involved),
Table 3. Short-Term Outcomes of Cases

\begin{tabular}{ll}
\hline Characteristic & Patients $(\mathbf{n}=\mathbf{7 5})$ \\
\hline Major adverse cardiovascular events & \\
Cardiac death & $2(2.67 \%)$ \\
Cardiogenic shock & $0(0 \%)$ \\
Left ventricular failure & $1(1.33 \%)$ \\
Recurrent myocardial infraction & $0(0 \%)$ \\
Hemodynamically unstable arrhythmia & $1(1.33 \%)$ \\
Prolonged angina pain & $0(0 \%)$ \\
End outcomes & \\
In-hospital mortality & $2(2.67 \%)$ \\
30-day mortality & $0(0 \%)$ \\
\hline
\end{tabular}

Values are expressed as number (percent).

although its effect on other angiographic outcomes (number of involved coronary vessels) is yet to be determined. Although sd-LDL levels display a positive correlation with demographic characteristics (age and BMI), diagnosis, and risk factors, further studies are warranted to establish the significant correlation between sd-LDL and these outcomes.

\section{Acknowledgments}

None to declare.

\section{Financial Disclosure}

None to declare.

\section{Conflict of Interest}

None to declare.

\section{Informed Consent}

All patients provided written informed consent.

\section{Author Contributions}

Abhishek Singh, Rajendra Puhan and Akshyaya Pradhan conceptualized and designed the study. All authors acquired and interpreted the data. Akshyaya Pradhan wrote the manuscript. All authors revised and approved the final manuscript.

\section{Data Availability}

Any inquiries regarding supporting data availability of this 
study should be directed to the corresponding author.

\section{Abbreviations}

ACS: acute coronary syndrome; BMI: body mass index; CAD: coronary artery disease; HDL: high-density lipoprotein; lb-LDL: large buoyant LDL; LAD: left anterior descending coronary artery; LBBB: left bundle branch block; LDL: low-density lipoprotein; sd-LDL: small density low-density lipoprotein; MACE: major adverse cardiac events; NSTEMI: non-ST-segment elevation myocardial infarction; STEMI: STsegment elevation myocardial infarction; UA: unstable angina

\section{References}

1. Emadzadeh MR, Alavi MS, Soukhtanloo M, Mohammadpour T, Rahsepar AA, Tavallaie S, Khojasteh R, et al. Changes in small dense low-density lipoprotein levels following acute coronary syndrome. Angiology. 2013;64(3):216-222.

2. Nishikura T, Koba S, Yokota Y, Hirano T, Tsunoda F, Shoji M, Hamazaki Y, et al. Elevated small dense lowdensity lipoprotein cholesterol as a predictor for future cardiovascular events in patients with stable coronary artery disease. J Atheroscler Thromb. 2014;21(8):755-767.

3. Yildirim E, Bugan B, Celik M, Yuksel UC, Yalcinkaya E. Small dense low-density lipoprotein could be used as a therapeutic marker for treatment in patients with acute coronary syndrome. Angiology. 2013;64(8):644.

4. Hirano T, Ito Y, Koba S, Toyoda M, Ikejiri A, Saegusa $\mathrm{H}$, Yamazaki J, et al. Clinical significance of small dense low-density lipoprotein cholesterol levels determined by the simple precipitation method. Arterioscler Thromb Vasc Biol. 2004;24(3):558-563.

5. Fukushima Y, Hirayama S, Ueno T, Dohi T, Miyazaki T, Ohmura H, Mokuno H, et al. Small dense LDL cholesterol is a robust therapeutic marker of statin treatment in patients with acute coronary syndrome and metabolic syndrome. Clin Chim Acta. 2011;412(15-16):1423-1427.

6. Wu B, Yu Z, Tong T, Tong X, Yang Y, Tang Y, Ren H, et al. Evaluation of small dense low-density lipoprotein concentration for predicting the risk of acute coronary syndrome in Chinese population. J Clin Lab Anal. 2020;34(3):e23085.

7. Haffner SM, D'Agostino R, Jr., Goff D, Howard B, Festa A, Saad MF, Mykkanen L. LDL size in African Americans, Hispanics, and non-Hispanic whites : the insulin resistance atherosclerosis study. Arterioscler Thromb Vasc Biol. 1999;19(9):2234-2240.

8. Enkhmaa B, Anuurad E, Berglund L. Lipoprotein (a): impact by ethnicity and environmental and medical conditions. J Lipid Res. 2016;57(7):1111-1125.

9. Cho Y, Kim Y, Kim JH, Jee SH, Han K. The plasma small dense LDL-cholesterol calculation formula proposed by Srisawasdi et al is not applicable to Koreans who are healthy or have metabolic syndrome. Am J Clin Pathol. 2012;138(5):754-755; author reply 756 .
10. Goel PK, Ashfaq F, Khanna R, Ramesh V, Pandey CM. The association between small dense low density lipoprotein and coronary artery disease in North Indian patients. Indian J Clin Biochem. 2017;32(2):186-192.

11. Moon JY, Kwon HM, Kwon SW, Yoon SJ, Kim JS, Lee SJ, Park JK, et al. Lipoprotein(a) and LDL particle size are related to the severity of coronary artery disease. Cardiology. 2007;108(4):282-289.

12. Kwon SW, Yoon SJ, Kang TS, Kwon HM, Kim JH, Rhee J, Lee SJ, et al. Significance of small dense low-density lipoprotein as a risk factor for coronary artery disease and acute coronary syndrome. Yonsei Med J. 2006;47(3):405414.

13. Koba S, Hirano T, Ito Y, Tsunoda F, Yokota Y, Ban Y, Iso $\mathrm{Y}$, et al. Significance of small dense low-density lipoprotein-cholesterol concentrations in relation to the severity of coronary heart diseases. Atherosclerosis. 2006;189(1):206-214.

14. Sakai K, Koba S, Nakamura Y, Yokota Y, Tsunoda F, Shoji M, Itoh Y, et al. Small dense low-density lipoprotein cholesterol is a promising biomarker for secondary prevention in older men with stable coronary artery disease. Geriatr Gerontol Int. 2018;18(6):965-972.

15. Jin JL, Zhang HW, Cao YX, Liu HH, Hua Q, Li YF, Zhang $\mathrm{Y}$, et al. Association of small dense low-density lipoprotein with cardiovascular outcome in patients with coronary artery disease and diabetes: a prospective, observational cohort study. Cardiovasc Diabetol. 2020;19(1):45.

16. Toft-Petersen AP, Tilsted HH, Aaroe J, Rasmussen K, Christensen T, Griffin BA, Aardestrup IV, et al. Small dense LDL particles - a predictor of coronary artery disease evaluated by invasive and CT-based techniques: a case-control study. Lipids Health Dis. 2011;10:21.

17. Gardner CD, Fortmann SP, Krauss RM. Association of small low-density lipoprotein particles with the incidence of coronary artery disease in men and women. JAMA. 1996;276(11):875-881.

18. Koba S, Hirano T, Yoshino G, Sakai K, Sakaue T, Adachi M, Katagiri T. Remarkably high prevalence of small dense low-density lipoprotein in Japanese men with coronary artery disease, irrespective of the presence of diabetes. Atherosclerosis. 2002;160(1):249-256.

19. Katzel LI, Coon PJ, Rogus E, Krauss RM, Goldberg AP. Persistence of low HDL-C levels after weight reduction in older men with small LDL particles. Arterioscler Thromb Vasc Biol. 1995;15(3):299-305.

20. Hirayama S, Soda S, Ito Y, Matsui H, Ueno T, Fukushima $\mathrm{Y}$, Ohmura H, et al. Circadian change of serum concentration of small dense LDL-cholesterol in type 2 diabetic patients. Clin Chim Acta. 2010;411(3-4):253-257.

21. Kumar N, Varghese L, Chacko ST, Karuppusai R, Jose A, Joseph G. Looking beyond LDL-cholesterol-A study on extended lipid profile in Indian patients with acute coronary syndrome. J Clin Diagn Res. 2019;13(11):1-6.

22. Mittal A, Gupta MD, Meennahalli Palleda G, Vyas A, Tyagi S. Relationship of plasma adiponectin levels with acute coronary syndromes and coronary lesion severity in north Indian population. ISRN Cardiol. 2013;2013:854815.

23. Patange A, Immadisetty S, Vora H, Mankodia H. Lipid 
levels in acute coronary syndromes admitted in medical intensive care unit in a tertiary care hospital. J Evol Med Dent Sci. 2020;9(8):520-523.

24. Anjana RM, Pradeepa R, Deepa M, Datta M, Sudha V,
Unnikrishnan R, Nath LM, et al. The Indian Council of Medical Research-India Diabetes (ICMR-INDIAB) study: methodological details. J Diabetes Sci Technol. 2011;5(4):906-914. 